\title{
Investigation of the Periodic Magnetic Field Modulation in LHC Superconducting Dipoles
}

\author{
P. Pugnat, Th. Schreiner, and A. Siemko
}

\begin{abstract}
The windings of high-field accelerator magnets are usually made of Rutherford-type superconducting cables. The magnetic field distribution along the axis of such magnets exhibits a periodic modulation with a wavelength equal to the twist pitch length of the cable used in the winding. This effect, resulting from quasipersistent currents, was investigated with a Hall probes array inserted inside the aperture of the LHC superconducting dipoles, both in short models and full-scale prototypes. The amplitude and the time dependence of this periodic field oscillation have been studied as a function of the magnet current history. The origin and the impact on the LHC dipoles stability of the nonuniform current redistribution producing such a field modulation are discussed.
\end{abstract}

Index Terms-LHC, periodic field pattern, persistent eddy-current, quench performance, Rutherford type cable, superconducting magnets.

\section{INTRODUCTION}

W HEN an accelerator magnet made of multistrand superconducting cable is energized, an axial periodic modulation of the magnetic field is established with a wavelength equal to the cable transposition pitch length. This Periodic Field Pattern (PFP) was first discovered in HERA dipoles in 1991 and was found to be present in all normal and skew harmonic components of the field [1]. The study of this phenomenon, its time decay and related effects are of importance for the field quality requirements for the Large Hadron Collider (LHC) [2] as well as for the magnet quench performance especially in real operating conditions of the machine. The PFP originates from nonuniform interstrand current distribution [3] which can lower significantly the current margin of the superconducting cable. This report mainly focuses on the latter aspect and provides a discussion concerning the origin and the consequence of such induced current on the quench performance of the LHC dipoles.

\section{EXPERIMENTAL}

\section{A. General Layout of the Test Stations}

The investigations of the PFP have been carried out on short dipole models [4] as well as on full-scale prototypes and first pre-series cryo-dipoles for the LHC [5]. To study both these types of superconducting magnet of different scale, two types of cryogenic test facilities have been used at CERN.

Manuscript received September 24, 2001.

P. Pugnat and A. Siemko are with the European Laboratory for Nuclear Research, CERN, Geneva 23, CH-1211, Switzerland (e-mail: \{Pierre.Pugnat; Andrzej.Siemko\}@ cern.ch).

Th. Schreiner is with the European Laboratory for Nuclear Research, CERN, Geneva 23, CH-1211, Switzerland and is also with the University of Technology of Vienna (e-mail: Thomas.Schreiner@cern.ch).

Publisher Item Identifier S 1051-8223(02)03471-1.
1) Cryogenic Test Facilities for Short Superconducting Magnets: The measurements of the PFP inside short models have been performed at the Block- 4 cryogenic test facilities also used to characterize the protection diodes and the corrector magnets for the LHC [6]. In this test station, two vertical set-up can be used to suspend and immerse 1-meter long magnets in superfluid helium. Inside the cryostats, the so-called $\lambda$-plate separates the pool of boiling helium bath from the superfluid one, both maintained at atmospheric pressure (Claudet bath). The subcooled superfluid helium in the lower portion of the cryostat is achieved with a heat exchanger where saturated superfluid conditions are obtained from the Joule-Thomson expansion of the liquid helium. The $\lambda$-plate has a number of leaktight feedthroughs for superconducting busbars, instrumentation wires and sliding bearing for the rotating shaft used for measurements of the field quality. To make measurements of the PFP, a special shaft was equipped with stainless-steel boxes containing Hall plates. This shaft was installed inside the magnet cold bore and was immersed inside the superfluid helium during the measurements.

2) Superconducting Magnet Test Plant for Full-Scale LHC Cryo-Magnets: Full-scale LHC magnets are of "embarked cryogenics" type. They should be first assembled into cryo-dipoles before to be tested at the CERN Superconducting Magnet Test Plant (SMTP) [5]. At the SMTP, cryo-dipoles are connected to the Cold Feed Boxes (CFB) which provide superfluid liquid helium and contain current leads to power the coils. On the test bench, the cryo-dipoles are filled with around 3001 of liquid helium. This constitutes in normal operation the static bath of pressurized superfluid helium through which heat is transported by conduction to a linear cold source made of a heat exchanger tube threaded through the whole cold mass. In this heat exchanger, the heat is absorbed quasiisothermally by gradual vaporization of flowing saturated superfluid helium. On the test bench, both apertures of the cryo-dipole are equipped with anticryostats. They allow to insert different types of equipment operating at room temperature like rotating shafts for standard magnetic field measurements or Hall probe set-ups for more local investigations.

\section{B. Hall Probe Measurement Systems}

Because of the small size of their sensitive area and their relative high sensitivity, Hall probes are ideal devices to measure the PFP inside narrow apertures of accelerator superconducting magnets.

1) Hall Probes Set-Up to Study the Magnetic Field of Short Superconducting Magnets: Up to thirteen cryogenic Hall probes of three different types, provided by the manufacturer 
TABLE I

Characteristics of Hall Probes

\begin{tabular}{lccc}
\hline $\begin{array}{l}\text { Hall Probes } \\
\text { Type }\end{array}$ & $\begin{array}{l}\text { Sensitivity in } \\
\text { V/(T A) }\end{array}$ & $\begin{array}{c}\text { Sensitive } \\
\text { area in } \text { mm }^{2}\end{array}$ & $\begin{array}{l}\text { Number } \\
\text { of units }\end{array}$ \\
\hline HHP-NP $^{\mathrm{a}}$ & 2.308 & $1.25 \times 0.5$ & 1 \\
LHP-MP $^{\mathrm{a}}$ & $0.275-0.44$ & $0.1 \times 0.1$ & 3 \\
HHP-MU $^{\mathrm{a}}$ & $4.66-5.11$ & $0.1 \times 0.1$ & 9 \\
HHP-NU $^{\mathrm{b}}$ & $4.44-4.56$ & $1.25 \times 0.5$ & 6 \\
\hline used in cold conditions inside LHC short models & \\
b used at room temperature inside full-scale LHC dipoles
\end{tabular}

TABLE II

LHC Short Dipole Models, Full-Scale Prototypes and FirST PRESERIES DiPOLES WHERE THE PFP WAS MEASURED

\begin{tabular}{ll}
\hline Magnet Name & Main characteristics \\
\hline MBSMS17_V3 & $\begin{array}{l}\text { Single aperture model, 1-meter long, 6-block } \\
\text { variant design, aluminium collar. }\end{array}$ \\
MBSMT4_V2 & $\begin{array}{l}\text { Twin apertures model, 1-meter long, 6-block } \\
\text { baseline design, stainless steel collar. }\end{array}$ \\
MBSMT4_V5 & $\begin{array}{l}\text { Twin apertures model, 1-meter long, 6-block } \\
\text { baseline design, stainless steel collar, } \\
\text { ferromagnetic yoke centred. }\end{array}$ \\
MBSMT5_V3 & $\begin{array}{l}\text { Twin apertures model, 1-meter long, 6-block } \\
\text { baseline design, plastic \& stainless steel collar. }\end{array}$ \\
MBP2O1 & $\begin{array}{l}\text { 15-meter long prototype dipoles of the last } \\
\text { generation, 6-block baseline design, stainless steel }\end{array}$ \\
MBP2A2 & collar. \\
MBP2O2 & $\begin{array}{l}\text { First and third 15-meter long pre-series dipole, } \\
\text { 6-block baseline design, stainless steel collar. }\end{array}$ \\
MBPSO01 &
\end{tabular}

AREPOC have been used in the study performed on short magnet models. Their main characteristics are listed in Table I. The behavior of each Hall probe type was investigated at $1.9 \mathrm{~K}$ [7]. In particular the so-called de Haas-van Alphen oscillations were characterized. For the measurements of the PFP in the normal operation mode, Hall probes were connected in series and the input current value was fixed to $5 \mathrm{~mA}$.

The Hall probes were mounted inside a stainless steel case and fixed on a special rotating shaft. The probes were located inside the magnet aperture at a radius of $17 \mathrm{~mm}$ and covered the central part of short dipole model over a length of $200 \mathrm{~mm}$. They allow measurement of the radial component of the total magnetic field as a function of azimuthal angle for different positions along the magnet axis. Only results obtained for orientation of the Hall probes sensitive area perpendicular to the main dipole field direction will be presented.

2) Hall Probes Set-Up to Study the Magnetic Field of Full-Scale LHC Dipoles: For the measurements of the PFP inside long LHC cryo-dipoles, six Hall probes also provided by AREPOC have been used (Table I). They were mounted on two rings at a radius of $17 \mathrm{~mm}$ on the set-up dedicated for the snap-back study in LHC dipoles [8]. Hall probes were connected in series and the input current value was fixed to $50 \mathrm{~mA}$. The results presented in this article concern the measurements performed with the two probes perpendicular to the main field direction and distant of $55 \mathrm{~mm}$. The magnetic field inside the dipole apertures has been scanned by moving the Hall probes set-up axially at fixed orientation.

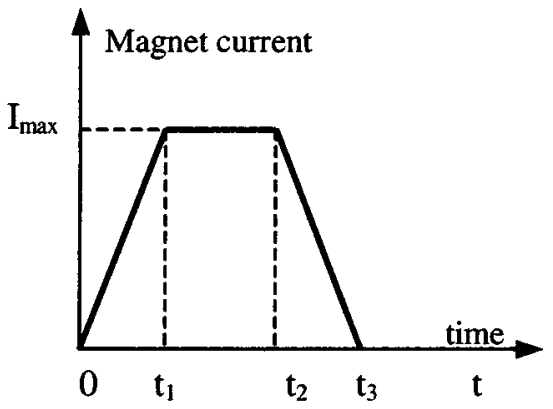

Fig. 1. Example of current cycle performed.

3) Measurement and Acquisition Systems: In both test stations, KEITHLEY 2001 digital multimeters were used to measure the Hall voltage and the current inside the magnet. Dedicated LabVIEW ${ }^{\circledR}$ software running on a SUN workstation, were used to interface with the digital multimeters via a GPIB bus for control, data acquisition and storage.

\section{Magnets Tested}

The PFP was investigated inside the aperture of several short model and long prototype superconducting dipoles (Table II) built mainly to study and to improve the mechanical structural stability and the field quality given by the design of the LHC main dipoles [4], [5].

\section{MEASUREMENTS, RESUlTS AND ANALYSIS}

\section{A. Measurements}

The superconducting magnets were submitted to current cycles of the type shown in Fig. 1, with different values of the maximum current $\left(I_{\max }\right)$ and times $t_{1}, t_{2}$ and $t_{3}$.

The local magnetic field inside the magnet aperture was measured with the Hall probes as a function of time during the flat-top and at the end of the current cycle.

\section{B. Study of the PFP as a Function of the Current Cycle}

The PFP was clearly observed after the end of the current cycles for all magnets which were investigated when the flat-top duration $t_{2}-t_{1}$ was larger than $3000 \mathrm{~s}$. It was in general difficult to identify it on the current plateau (i.e., between time $t_{1}$ and $t_{2}$ ) mainly because of the contribution of other sources of field inhomogeneity such as the ferromagnetic yoke laminations. The PFP can have a very long decay time and was observed up to 81 hours after the end of the current cycle.

Two examples of PFP measured on the short magnet model MBSMS17_V3 at the time $t_{3}$ are given in Fig. 2. They correspond to a current cycle with a ramp-up to $9 \mathrm{kA}$ at $40 \mathrm{~A} / \mathrm{s}$ followed by a ramp-down at $-40 \mathrm{~A} / \mathrm{s}$ after a plateau duration of $500 \mathrm{~s}$ and $3000 \mathrm{~s}$, respectively. Both these PFPs can be well approximated by the relation:

$$
B(z, t)=B_{0}(t)+B_{1}(t) \sin (2 \pi z / \lambda+\phi)
$$

with the fitting parameters given in Table III.

The $\lambda$ value in (1) is found (Table III) to be close to the twist pitch length of the cable used for the inner layer of the coil. 


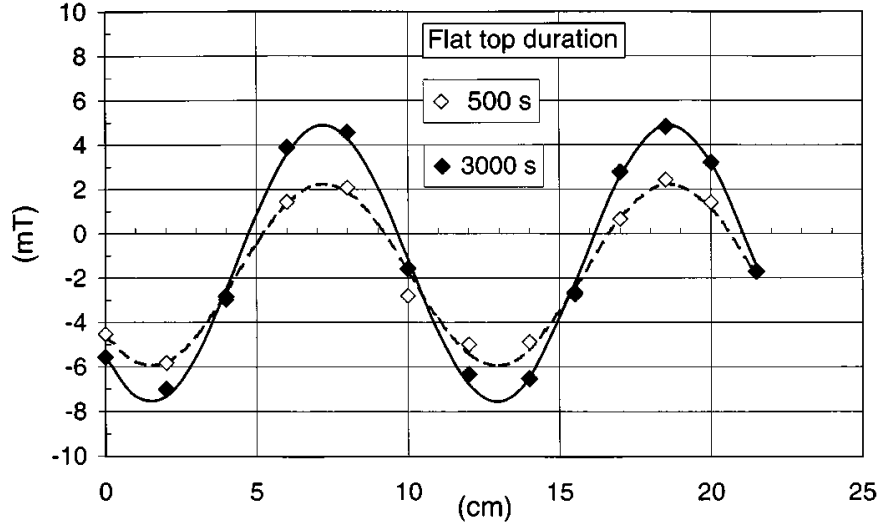

Fig. 2. Example of two periodic field patterns measured in the short model MBSMS17_V3 at the time $t_{3}$ of the current cycle shown in Fig. 1.

This result suggests a nonuniform interstrand current distribution inside the superconducting cable of the coil [3]. In order to understand the origin of such nonuniformity, previous studies have identified two main mechanisms. The first one concerns the spatial variation of the time derivative of the magnetic field along the cable [9], [10]. The second one is related to the variation of the cross contact resistance $R_{c}$ between strands of the cable [10].

The homogeneous term of (1) obtained from the fit of the PFPs measured at $t_{3}, B_{0}\left(t_{3}\right)$, is negative (Table III) because it comes mainly from the remanent magnetization of superconducting filaments. Concerning the amplitudes of the PFP at time $t_{3}, B_{1}\left(t_{3}\right)$, it is found to increase with the flat-top duration (Fig. 2). This result can be understood qualitatively within the framework of existing theoretical models [3], [9] and [10]. During the ramp-up of the current cycle (i.e., up to $t_{1}$ ), the Rutherford type superconducting cable charges itself with a nonuniformly distributed current wave. On the plateau of the cycle this wave diffuses slowly and its amplitude decays, in other words the current tends to be shared more uniformly between strands. During the ramp-down, the superconducting cable charges itself with a nonuniformly distributed current wave of an opposite sign as compared to the current ramp-up (antiwave). As a consequence, the PFP measured at the time $t_{3}$ results from the composition of two waves, which can partially cancel. The amplitude of the resulting PFP $B_{1}\left(t_{3}\right)$ increases with the flat-top duration because the compensation effect between the wave and the antiwave become less effective.

More generally, the amplitudes $B_{1}\left(t_{1}\right)$ and $B_{1}\left(t_{3}\right)$ of the PFP depend strongly on the current cycle performed. For example, they are found to increase exponentially with the flat top duration like the voltage of a charging capacitor. In the case of an asymmetric cycle with a ramp-down much faster than the ramp-up, the PFP exhibits a larger amplitude $B_{1}\left(t_{3}\right)$ as compared to a symmetric cycle. This effect can also be explained by a lower compensation effect between the two eddy current waves. Another result obtained is that the amplitude of PFP, $B_{1}\left(t_{1}\right)$, is found to increase significantly with the maximum current of the cycle. As an example, the amplitude of the periodic pattern measured on the flat-top inside the aperture of MBSMS17_V3 reached $32 \mathrm{mT}$ at $13 \mathrm{kA}$ whereas it was only equal to $16.8 \mathrm{mT}$ when the current cycle is stopped at $9 \mathrm{kA}$ [11].
TABLE III

FitTing Parameters of MEASURed Data ShOWN IN Fig. 2 USING THE RELATION (1)

\begin{tabular}{lcc}
\hline $\begin{array}{l}\text { Fitting } \\
\text { Parameters }\end{array}$ & $\begin{array}{l}\text { Current cycle with flat } \\
\text { top time of } \mathbf{5 0 0} \mathrm{s}\end{array}$ & $\begin{array}{l}\text { Current cycle with flat } \\
\text { top time of 3000 s }\end{array}$ \\
\hline $\mathrm{B}_{0}(\mathrm{mT})$ & -1.85 & -1.33 \\
$\mathrm{~B}_{1}(\mathrm{mT})$ & 4.09 & 6.23 \\
$\lambda(\mathrm{cm})$ & 11.4 & 11.4 \\
$\phi(\mathrm{rad})$ & -2.4 & -2.39 \\
\hline
\end{tabular}

TABLE IV

Results ObTained on SOME LHC Short Dipole Models, Full-Scale PROTOTYPES AND FIRST PRESERIES DIPOLES

\begin{tabular}{|c|c|c|}
\hline Magnet Name & $\begin{array}{l}\text { PFP Amplitude } B_{1} \\
\text { measured at } t=2500 \mathrm{~s} \\
\text { in } \mathrm{mT}\end{array}$ & $\begin{array}{l}\text { Cross contact } \\
\text { resistance } \mathbf{R}_{c} \\
\text { in } \mu \Omega\end{array}$ \\
\hline MBSMS17_V3 & 2.27 & $\mathrm{a}$ \\
\hline MBSMT4_V2 & 1.17 & a \\
\hline MBSMT4_V5 & 1.15 & a \\
\hline MBSMT5_V3 & 0.19 & a \\
\hline MBP2O1 & 0.16 & $40 \pm 2$ \\
\hline MBP2A2 & 0.13 & $70 \pm 7$ \\
\hline MBP2O2 & 0.18 & $42 \pm 2$ \\
\hline MBPSO01 & 0.15 & $63 \pm 2$ \\
\hline MBPSA01 & 0.25 & $37 \pm 2$ \\
\hline
\end{tabular}

\section{Study of the PFP Inside Long and Short Dipoles}

All superconducting dipoles were submitted to the so-called current reference cycle with a ramp-up to $9 \mathrm{kA}$ at $40 \mathrm{~A} / \mathrm{s}$ followed by a ramp-down at $-40 \mathrm{~A} / \mathrm{s}$ after a plateau duration of $1000 \mathrm{~s}$. The amplitudes of the PFP deduced from the fit of the measured data using (1) are listed in the Table IV.

The first conclusion which can be drawn is that no clear distinction can be observed between the PFP amplitudes measured in long and in short dipoles. In addition, the largest spread in $B_{1}$ values is observed inside short dipole models whereas all results obtained in long dipoles are very close. This result can be explained by the oxidation process of the strand coating ( $\mathrm{SnAg}_{5} \%$ wt. $)$ developed at CERN for the superconducting cables of the 3rd generation prototypes, pre-series and series magnets [12].

For long dipoles, the so-called "Field-Advance" produced by eddy-current was also measured with Hall probes during current cycles optimized for this study [13]. The values of the cross-contact resistance $R_{c}$ deduced from such measurements are also reported in Table IV. These results allow to plot the dependence of the PFP amplitude as a function of $1 / R_{c}$ (Fig. 3). As expected [9], [10], the data can be fitted with a linear variation crossing the origin. The slope of this straight line will allow to estimate the amplitude of the nonuniform current distribution assuming the worst case (see next paragraph) for the LHC superconducting dipoles.

\section{EFFECT OF THE NON-UNIFORM INDUCED CURRENT ON THE QUENCH PERFORMANCE OF LHC DIPOLES}

The oxidation process of the strand coating developed at CERN for the superconducting cables for the LHC is expected to guarantee a minimum value for $R_{c}$ equal to $20 \mu \Omega$ [12]. This is actually the case as it can be seen in Table IV for $R_{c}$ values 


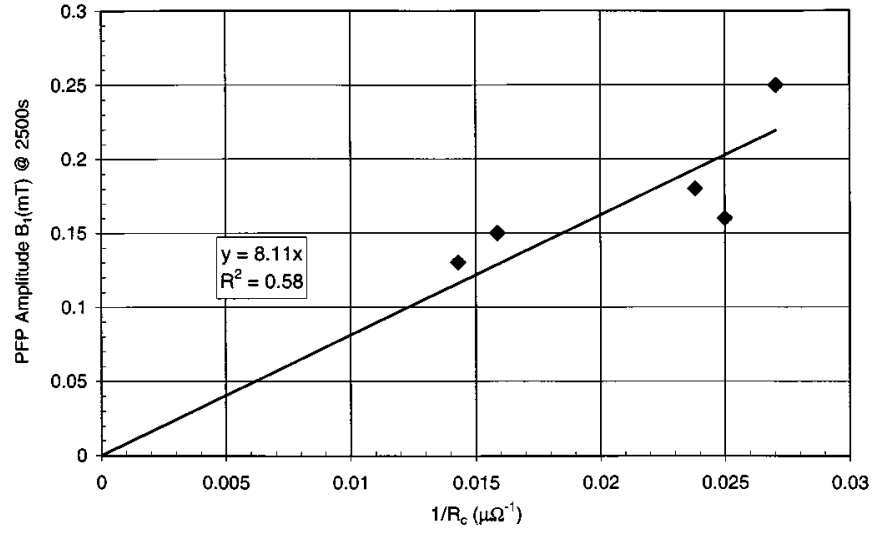

Fig. 3. PFP amplitude obtained after a reference cycle versus $1 / R_{c}$ deduced from field-advance measurements.

deduced from measurements performed on prototypes and first pre-series dipoles. According to the linear fit of Fig. 3, a $R_{c}$ value of $20 \mu \Omega$ corresponds to an amplitude for the PFP equal to $0.41 \mathrm{mT}$. The study of the PFP performed on short models gives typically a factor around five between the amplitudes of the PFP taken at the time $t_{1}$ and $t_{3}$ of the current cycle up to the nominal value shown in Fig. 1 [11]. As a consequence, for LHC dipole magnets with the lowest cross contact resistance expected, the PFP amplitude can reach $2 \mathrm{mT}$ at the nominal field. An estimate of the current imbalance between strands required to produce such a PFP amplitude is around $\pm 22 \mathrm{~A}$ for the case of the LHC dipole final design. This value can be compared to the current margin at the nominal field of half of the cable which is around $1000 \mathrm{~A}$. Moreover for the real operation of the LHC machine such a margin will be drastically reduced due to beam losses. As a consequence, nonuniform superimposed induced currents may provoke premature quenches of the dipoles with the lowest $R_{c}$ values but the instability with respect to quench performance provoked by the PFP is much less critical than anticipated from the worst case obtained with short dipole models [11].

\section{CONCLUSION}

This study performed on LHC superconducting dipoles of two different scales has shown the lack of correlation between the PFP amplitude and the length of the magnet. The dependence of nonuniform current distribution on local characteristics such as the cross contact resistance was observed. The PFP can affect the stability of LHC main dipoles with respect to quench performance. This problem would have been more serious if the cross contact resistance between strands had not been controlled.

\section{ACKNOWLEDGMENT}

The authors would like to thank A. A. Akhmetov for fruitful discussions, the Block-4 and the SM-18 operation teams for their devotion during the tests of magnets, and J. Billan and D. Tommasini for their help and support during the preparation of the experimental investigations.

\section{REFERENCES}

[1] H. Brück and D. Gall et al., "Observation of a period pattern in the persistent-current fields of the superconducting Hera magnets," in Proc. $P A C$ '91, San Francisco, May 1991.

[2] L. Bottura, L. Walckiers, and R. Wolf, "Field errors decay and snap-back in LHC model dipoles," in Proc. ASC'96, Pittsburgh, August.

[3] A. A. Akhmetov, A. Devred, R. G. Mints, and R. I. Schermer, "Current loop decay in Rutherford-type cable," in Supercollider 5, P. Hale, Ed. New York: Plenum Press, 1994, pp. 443-446.

[4] D. Tommasini et al., "Status of the short dipole model program and feedback for the series design,", submitted for publication.

[5] L. Bottura, G. D’Angelo, M. Gateau, P. Legrand, M. Modena, K. Naoui, D. Perini, P. Pugnat, S. Sanfilippo, F. Savary, W. Scandale, A. Siemko, P. Sievers, G. Spigo, J. Vlogaert, and C. Wyss, "Performance of the LHC final prototype and first pre-series superconducting dipole magnets,", submitted for publication.

[6] W. Venturini Delsolaro, A. Arn, L. Bottura, C. Giloux, R. Mompo, A. Siemko, and L. Walckiers, "The test facility for the short prototypes of the LHC superconducting magnets," in CEC/ICMC 2001, submitted for publication.

[7] P. Pugnat and A. Siemko, "Calibration and dynamic response of Hall probes at low temperature for current sharing experiment,", CERN-LHC-MTA Internal Note 98-034, 1998.

[8] L. Bottura, L. Larsson, S. Schloss, M. Schneider, and N. Smirnov, "A fast sextupole probe for snapback measurement in the LHC dipoles," IEEE Trans. Appl. Superconduct., vol. 10, no. 1, pp. 1435-1438, 2000.

[9] L. Krempasky and C. Schmidt, "Theory of supercurrents and their influence on field quality and stability of superconducting magnets," J. Appl. Phys., vol. 78, pp. 5800-5810, 1995.

[10] A. P. Verweij, "Electrodynamics of superconducting cables in accelerator magnets," Ph.D. dissertation, Twente University, 1995.

[11] P. Pugnat, Th. Schreiner, and A. Siemko, "Investigation of the periodic field modulation inside apertures of LHC superconducting dipole models," in Proc. EPAC'O0, Vienna, June 2000.

[12] J. Adam, T. Boutboul, G. Cavallari, Z. Charifoulline, C.-H. Denarie, S. Le Naour, D. F. Leroy, L. R. Oberli, D. Richter, A. P. Verweij, and R. Wolf, "Status of the LHC superconducting cables mass production,", submitted for publication.

[13] A. Akhmetov, S. Amet, I. Balaazi, L. Bottura, M. Gateau, P. Pugnat, and L. Walckiers, "Loss and dynamic magnetic field measurements in LHC dipoles,", submitted for publication. 